\title{
Interferencias oclusales asociadas a maloclusiones funcionales en dentición mixta
}

Occlusal Interferences Associated with Functional Malocclusions in Mixed Dentition
Interferências Oclusais Associadas Às Maloclusões Funcionais Na Dentição Mista
Paola B. Olivera

Asignatura Metodología de la Investigación.

Edna Y. Meza Asignatura Metodología de la Investigación.

María N. Rosende Asignatura Psicología del Niño, Adolescente y Adulto.

\section{María S. Dho}

Asignatura Práctica Clínica Preventiva I. Facultad de Odontología de la Universidad Nacional del Nordeste (UNNE).

Alina N. Peláez Asignatura Metodología de la Investigación.

\section{Autor de Correspondencia} Paola B. Olivera Facultad de Odontología. UNNE Av. Libertad 5450. Corrientes (Capital). República Argentina. Email:paolaolivera27@gmail.com

\section{Resumen}

La existencia de interferencias oclusales durante el desarrollo de la dentición, sin un diagnóstico precoz y rápido tratamiento de la anomalía pueden producir trastornos serios, que generalmente conducen a mordidas cruzadas funcionales, mordidas abiertas, así como mordida cruzada anterior. Determinar la prevalencia de interferencias oclusales y su asociación con maloclusiones funcionales en dentición mixta. Se realizó un estudio observacional descriptivo transversal. La población de estudio estuvo constituida por todos los pacientes que asistieron a la Clínica del Módulo Atención Integral del Niño y Adolescente de la Facultad de Odontología de la UNNE, para su atención integral en el período de los meses de abril a noviembre del año 20।8. En todos los pacientes que conformaron la muestra de estudio se evaluó con distintos parámetros clínicos la presencia de interferencias oclusales y maloclusiones funcionales. De los 49 pacientes evaluados, el $63 \%$ presentó interferencias oclusales, con una prevalencia de interferencias unilaterales de un $68 \%$ y bilaterales en un $32 \%$. Con respecto a las maloclusiones funcionales se presentaron en un $67 \%$ de los niños evaluados. Se puedo determinar asociación estadísticamente significativa $(p \leq 0,05)$ entre la presencia de interferencias oclusales y maloclusiones funcionales en pacientes pediátricos con dentición mixta. Se halló una alta prevalencia de interferencias oclusales, asociadas a maloclusiones funcionales en niños con dentición mixta. 
REVISTAFACULTAD DE ODONTOLOGÍA ISSN 1668-7280 / ISSN-E 2683-7986

10
INVESTIGACIÓN

Olivera - Meza - Rosende - Dho - Peláez
Palabras Claves: Disfunción temporomandibular, Niños, Oclusión.

\section{Abstract}

The existence of occlusal interferences during the development of the dentition, without an early diagnosis and rapid treatment of the anomaly can cause serious disorders. They generally lead to functional crossbites, open bites, as well as anterior crossbite. The aim of this study was to determine the prevalence of occlusal interferences and their association with functional malocclusions in mixed dentition. A transversal descriptive observational study was carried out. The study population consisted of all the patients who attended the Clinic of Comprehensive Care of the Child and Adolescent Module of the School of Dentistry of the UNNE, for its comprehensive care during the period from April to November of the year 2018 . The presence of occlusal interferences and functional malocclusions was evaluated with different clinical parameters in all the patients that made up the study sample. Out of 49 patients evaluated, $63 \%$ presented occlusal interferences, within them $68 \%$ presented unilateral interference and $32 \%$ presented bilateral interference. We can determine a statistically significant association $(p \leq 0.05)$ between the presence of occlusal interferences and functional malocclusions in pediatric patients with mixed dentition. A high prevalence of occlusal interferences associated with functional malocclusions was found in children with mixed dentition.

Key Words: Temporomandibular Dysfunction, Children, Occlusion.

\section{Resumo}

A existência de interferência oclusal durante o desenvolvimento dos dentes, sem um diagnóstico precoce e tratamento rápido de anormalidade pode causar distúrbios graves, que geralmente levam à mordida cruzada funcional, mordida aberta e cruzada. $O$ objetivo deste estudo foi determinar a prevalência de interferências oclusais e sua associação com más oclusões funcionais na dentição mista. Foi realizado um estudo observacional descritivo transversal. A população do estudo foi constituída por todos os pacientes que compareceram à Clínica do Módulo Atendimento Integral das Crianças e Adolescentes na Faculdade de Odontologia da Universidade Nacional do Nordeste (UNNE), para o cuidado integral no período de abril a novembro de 2018. Em todos os pacientes que conformaram a amostra foi avaliada com diferentes parâmetros clínicos a presença de interferências oclusais e má oclusões. Dos 49 doentes avaliados, $63 \%$ tinha interferências oclusais, com uma prevalência de interferência unilateral de $68 \%$ e acordos bilaterais em $32 \%$. Quanto às más oclusões funcionais, elas foram encontradas em $67 \%$ das crianças avaliadas. Podemos determinar uma associação estatisticamente significativa $(p \leq 0,05)$ entre a presença de interferências oclusais e màs oclusões funcionáis.

Palavras chave: Disfunção Temporomandibular, Crianças, Oclusão.

\section{Introducción}

La dentición temporal al igual que la dentición mixta está sujeta a grandes modificaciones; mantener un control periódico permite prevenir e interceptar alteraciones que puedan afectar el normal funcionamiento del sistema neuromuscular y articular. Durante el desarrollo de la dentición es muy común encontrar alteraciones en la posición de los dientes, que condicionan desarmonías oclusales'. Un problema frecuente en la dentición infantil son las interferencias oclusales que pueden generar maloclusiones funcionales tales como mordidas abiertas anteriores, desviaciones de la línea media, mordidas cruzadas anteriores y posteriores ${ }^{2}$. Las interferencias oclusales si no son controladas precozmente pueden producir una desviación de la mandíbula en sentido anteroposterior o transversal. Lateralmente esta se desviará hacia el lado de la mordida invertida, lo que ocasiona falta de estímulo para el desarrollo del maxilar; discrepancia de la línea media inferior, una asimetría del arco dental y facial, con desviación lateral del mentón, hacia el lado cruzado y afectación del balance bilateral de los músculos masticatorios ${ }^{3}$. 
Todo esto crea movimientos patológicos de masticación, que impide la abrasión de las caras oclusales y el establecimiento de un plano de oclusión equilibrado y balanceado 4 . Obstaculizan las relaciones que se establecen entre los arcos dentarios e impiden el trayecto armonioso de la mandíbula desde la posición postural hasta los movimientos de la dinámica mandibular.

Algunos estudios han reportado casos donde las interferencias oclusales estuvieron estrechamente relacionadas con mordidas abiertas y disfunciones de la articulación temporomandibular, señalando las interferencias oclusales como signos predictivos del desarrollo de estos trastornos ${ }^{5,6}$. La mordida cruzada funcional existe cuando un niño cierra en oclusión céntrica, choca con un contacto prematuro y desliza hacia una relación de mordida cruzada. En diferentes estudios se menciona que esta alteración es una maloclusión común en la dentición temporaria y mixta ${ }^{7,8}$. El manejo de las mordidas cruzadas se debe iniciar en el momento en que se diagnostica, y preferiblemente en edades tempranas con el fin de tratarlas en el nivel primario de prevención, ya que al ser detectadas precozmente son fáciles de tratar por medio de procedimientos preventivos sencillos 9.10 .

La prevención y la atención debe ir dirigida especialmente a la dentición temporal o mixta temprana siendo esta privilegiada con respecto a otros grupos de edades, presentándose amplias posibilidades para desarrollar el trabajo clínico, y mejorar la oclusión en el adulto, ya que la dentición en estas edades, además de permitir la fonación y la alimentación del niño, servirá de guía en la erupción de los dientes permanentes, permitiendo el correcto desarrollo de las arcadas dentarias". El profesional debe ser capaz de diagnosticar tempranamente dichas anomalías en esta instancia, de suceder lo contrario, con el correr del tiempo se transformarán en serios trastornos de la oclusión que requerirán soluciones más complejas que podrían llegar hasta tratamientos quirúrgicos ${ }^{12,13}$.

Por todo lo expuesto el objetivo de este estudio fue determinar la prevalencia de interferencias oclusales y su asociación con maloclusiones funcionales en dentición mixta.

\section{Materiales y Métodos}

Se realizó un estudio observacional descriptivo transversal. La población de estudio estuvo constituida por todos los pacientes que asistieron a la Clínica del Módulo Atención Integral del Niño y Adolescente de la Facultad de Odontología de la UNNE, para su atención integral en el período de los meses de abril a noviembre del año 2018 , sobre los cuales se aplicaron los siguientes criterios de inclusión: pacientes con dentición mixta entre 6 y 12 años $y$ aquellos pacientes, padres y/o tutores que aceptaron formar parte del estudio disponiendo de su consentimiento para la utilización de los datos de exploración. Los criterios de Exclusión: pacientes que presentaron alguna patología sistémica significativa con deficiencia motriz o neurológica y aquellos pacientes que han recibido en el pasado, o estaban recibiendo en ese momento cualquier tipo de tratamiento ortopédico u ortodóncico. En base a estos criterios se conformó la Población Objeto constituida por 245 pacientes pediátricos; entre los que se seleccionaron 49 mediante un Muestreo al Azar Sistemático, estableciendo una muestra del $20 \%$ de la Población Objeto.

El examen clínico del paciente se llevó a cabo en las Clínicas de la Facultad de Odontología de la Universidad Nacional del Nordeste. Primero, se les presentó una hoja informativa a los pacientes, padres y/o tutores de los procedimientos y objetivos del trabajo, posteriormente firmaron un consentimiento informado. El examen clínico se realizó en sillones dentales, los cuales fueron efectuados por un único examinador entrenado a tal efecto. En todos los pacientes que conformaron la muestra de estudio se evaluó la presencia de interferencias oclusales y maloclusiones funcionales.

Para la evaluación clínica de Interferencias Oclusales se indicó al paciente que realice movimientos de apertura y cierre bucal para determinar la existencia de desviación mandibular; considerando interferencias oclusales al primer contacto que provocara un deslizamiento no fisiológico de la mandíbula, llevando a una $o$ ambas articulaciones fuera de su posición fisiológica (relación céntrica). En presencia de las interferencias oclusales se identificó si era unilateral o bilateral. De acuerdo con su 
REVISTA FACULTAD DE ODONTOLOGÍA

ISSN 1668-7280 / ISSN-E 2683-7986

12

localización, se clasificó en anterior o posterior.

En la evaluación clínica de maloclusiones funcionales se consideró a las mordidas abiertas, mordidas cruzadas y desviación de líneas medias. La mordida abierta anterior se determinó en los casos donde uno o más dientes anteriores no alcanzaran el plano de oclusión para hacer contacto con sus antagonistas. Se determinó con regla milimetrada y se clasificó en: mordida abierta moderada (apertura menor o igual a $3 \mathrm{~mm}$ ) y mordida abierta severa (más de $3 \mathrm{~mm}$ ). La mordida cruzada anterior se estableció cuando los incisivos superiores ocluyen por lingual de los incisivos inferiores, cuando estaba presente se especificó la distancia en milímetros. La mordida cruzada posterior, puede ser unilateral o bilateral, cuando las cúspides vestibulares de premolares o molares inferiores se encontraban más hacia vestibular que las cúspides bucales de los superiores; en presencia se especificó el número de dientes afectados. La desviación de la línea media se consideró cuando el punto de contacto mesial de incisivos centrales en la arcada superior, inferior o de ambas se alejaba hacia la derecha o izquierda del plano sagital medio. Cuando se hallaba presente, se especificó la arcada dentaria afectada, superior/inferior o ambas.

Se realizó un análisis descriptivo de los datos y para evaluar la asociación entre las interferencias oclusales y las maloclusiones funcionales, se realizó una prueba de independencia de Chi Cuadrado $\left(\mathbf{X}^{2}\right)$, con un nivel de significación del $5 \%(\alpha=0,05)$. El análisis de los datos se realizó mediante el software estadístico InfoStat (versión 2018) ${ }^{14}$.

\section{Resultados}

Los pacientes evaluados tenían un rango de edad de 6 a I I años, con un promedio de 7,45 \pm I, I9 años. De los 49 pacientes evaluados el $51 \%$ correspondió al sexo femenino y un $49 \%$ al sexo masculino.

El $63 \%$ del total de la muestra presentó interferencias oclusales, con una prevalencia de interferencias unilaterales de un $68 \%$ y bilaterales en un $32 \%$. La distribución de las interferencias fue mayor para el sector anterior en un $68 \%$ y $32 \%$ para el sector posterior.

Con respecto a las maloclusiones funcionales se presentaron en un $67 \%$ de los niños evaluados. La presencia de mordida abierta anterior fue de un $29 \%$, mientras que la mordida cruzada se encontró en un $27 \%$ de la población, distribuidas en el sector anterior en un $69 \%$ y posterior en un $31 \%$. La desviación de la línea media se pudo observar en un I8\% de los casos (Figura I). En la Tabla I, se presentan los resultados de la prueba de Independencia por $\left(X^{2}\right)$ entre Interferencias oclusales y las maloclusiones funcionales, donde se detecta asociación estadísticamente significativa $(p \leq 0,05)$ entre ambas variables.

\section{Discusión}

Los resultados obtenidos en el presente trabajo son similares a los valores encontrados por Cabrera Sánchez y col,' los cuales analizaron algunos factores capaces de influir en la aparición de interferencias oclusales en niños con dentición temporal y mixta en el área de salud de la Clínica Estomatológica Provincial Docente de Santiago de Cuba, donde las interferencias oclusales en 80 niños prevalecieron en un $66,2 \%$. Estos resultados difieren de los valores encontrados en el estudio de Cabrera, Pérez Varela y col., ${ }^{15}$ quienes hallaron en escuelas primarias urbanas de Ciudad de La Habana en niños con dentición mixta, que un 16,1 \% $(n=2893)$ de ellos presentó interferencias oclusales. Dichas diferencias podrían explicarse por el hecho que el presente estudio, al igual que el trabajo de Cabrera Sánchez y col.' se realizó en centros de atención odontológica, mientras que la investigación de Cabrera, Pérez Varela y col., ${ }^{15}$ se realizó en escuelas primarias.

Durante todo el desarrollo de la dentición es muy frecuente encontrar alteraciones en la posición de los dientes que conllevan a desarmonías oclusales. Un estudio realizado por UrregoBurbano y col., ${ }^{16}$ los cuales realizaron un estudio epidemiológico descriptivo en 436 pacientes con edades entre los 5 y los 12 años de edad, en el plano trasversal encontraron mordida cruzada completa en un 6,1 \%, presentándose en un $7,3 \%$ de manera unilateral y de manera bilateral 
Prevalencia de Interferencias Oclusales y Maloclusiones Funcionales en pacientes niños con dentición mixta

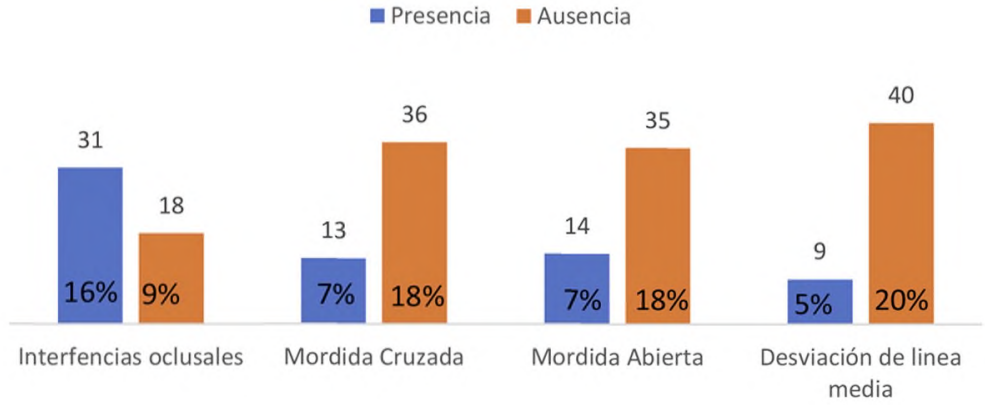

Figura I. Distribución de Interferencias oclusales y Maloclusiones Funcionales en niños con dentición mixta.

Tabla I. Valores obtenidos y sus correspondientes probabilidades, para la prueba de $\mathbf{X}^{2}$ de independencia entre interferencias oclusales y las maloclusiones funcionales en dentición mixta.

\begin{tabular}{|c|c|c|c|c|c|c|}
\hline \multirow{2}{*}{\multicolumn{2}{|c|}{$\begin{array}{l}\text { CARÁCTERÍSTICAS } \\
\text { CLÍNICAS }\end{array}$}} & \multicolumn{4}{|c|}{$\begin{array}{l}\text { MALOCLUSIONES } \\
\text { FUNCIONALES }\end{array}$} & \multirow{2}{*}{ TOTAL } \\
\hline & & Si & No & $x^{2}$ & P valor & \\
\hline \multirow{2}{*}{$\begin{array}{l}\text { INTERFERENCIAS } \\
\text { OCLUSALES }\end{array}$} & $\mathrm{Si}$ & 26 & 7 & \multirow[t]{2}{*}{10,48} & \multirow[t]{2}{*}{0,0012} & 33 \\
\hline & No & 5 & 11 & & & 16 \\
\hline \multicolumn{2}{|l|}{ TOTAL } & 31 & 18 & - & - & 49 \\
\hline
\end{tabular}

en un $2,4 \%$, valores bajos en comparación con los encontrados en este estudio.

Diferentes estudios, ${ }^{1,7}$ muestran la relación entre la presencia de maloclusiones funcionales y la existencia de interferencias oclusales como se expone en la presente investigación. Sus resultados muestran que estos problemas pueden ser diagnosticados y tratados por el estomatólogo general integral en el nivel primario de atención. En el ámbito estomatológico, se impone la necesidad de ampliar la visión oclusal hacia un entendimiento más funcional que morfológico, que lleve a una buena prevención, corrección y remisión de los pacientes con maloclusiones y esto solo es posible con un profundo entendimiento de las relaciones oclusales funcionales, en relación a esto, Suárez Gómez L y col. ${ }^{17}$ concluyen que las variables oclusales funcionales se asocian significativamente a las maloclusiones generales, por lo tanto es importante la prevención en el desarrollo de estas anomalías en denticiones primarias o mixtas.

\section{Conclusión}

En el presente estudio se identificó una alta prevalencia de interferencias oclusales en niños con dentición mixta, asociadas a maloclusiones funcionales. La presencia de mordidas abiertas fue la de mayor frecuencia, seguida de las mordidas cruzadas y por último la desviación de la línea media. Por todo ello se pudo determinar que es de suma importancia su detección precoz, para evitar alteraciones oclusales en la dentición permanente. 


\section{Referencias Bibliográficas}

I. Cabrera Sánchez TV, Martínez Ramos MR, Comas Mirabent R, González Esplanger L, Perú Seguí Y. Interferencias oclusales en niños con dentición temporal y mixta temprana. MEDISAN [Internet].2015 [citado 2016 Jun 03]; 19(3):321327. Disponible en:http://scielo.sld.cu/scielo.phpscript=sci_ arttext\&pid=S|02930| $920|5000300005 \&|$ ng=es.

2. Botero PM, Vélez N, Cuesta DP, Gómez E, Cossio M, Santos E. Profile of dental occlusion in children from Universidad Epidemiological Cooperativa de Colombia. Rev CES Odont. 2009; 22 (1): 9-13.

3. González Valdés D, Alemán Sánchez PC, Delgado Díaz Y, Díaz Ortega L, León López E. Presencia de mordida invertida en un grupo de niños y adolescentes. San Antonio de los Baños. 2014. Rev haban cienc méd [Internet]. 2015 [citado 2016 Jun 03]; 14 (3):270-280. Disponible en: http://scielo.sld.cu/scielo.php?script=sci_arttext\&pid=S|729 519X2015000300004\&lng=es.

4. Duque de Estrada Riverón J, Rodríguez Calzadilla A, Coutin Marie G, Riverón Herrera F. Factores de riesgo asociados con la enfermedad caries dental en niños, Rev Cubana Estomatol, Ciudad de La Habana, v. 40, n. 2, agosto 2003. Disponible en <http://scielo.sld.cu/scie-
lo.php?script=sci_arttext\&pid=S00347507200300020000 I \&lng=es\&nrm=iso >. accedido en 01 jul. 2019.

5. Dafabjanis P. Impact of nasal airway obstruction on dentofacial developmen and sep disturvance in children. J Clin Pediatr Dent. 2003; 27 (2): 95-100.

6. Louly F, Aranha Nouer PR, Janson G, Pinzan A. Dental arch dimensions in the mixed dentition: a study of Brazilian children from 9 to 12 years of age. J Appl Oral Sci. 201।;19 (2): 169-74.

7. Quintana Espinosa MT, Martínez Brito I. Interferencias oclusales y su relación con las maloclusiones funcionales en niños con dentición mixta. Rev Méd Electrón. 2010 [citado 02 jun 2016]; 32 (2). Disponible en: http://scielo.sld.cu/scielo.php?script=sci_ar ttext\&pid $=S|684| 824201000020000$

8. González Valdés D, Alemán Sánchez PC, Díaz Ortega L, Delgado Díaz Y. Alteraciones dentofaciales en niños de 6 a II años expuestos a factores de riesgo. Convención Internacional Estomatología 2010. [Citado 2016 may 27]. Disponible en: http://files. sld.cu/saludbucal/category/files/20I0.

9. Fróes $\mathrm{Cl}$, Silva $\mathrm{CS}$, Marques SB, Gondim VA. Prevalência de maloclusões em escolares de 6 a 12 anos na cidade de João Pessoa/Paraíba. Rev Bras Ci Saúde. 2009; 13 (2): 53-62. 
10. Beraud Osorio D, Sánchez MA, Murrieta JF, Mendoza VM. Prevalencia y factores de riesgo de mordida cruzada posterior en niños de 4-9 años de edad en ciudad $\mathrm{Ne}$ zahualcóyotl. Bol Med Hosp Infant Méx. 2004;6| (2): | $4 \mid$-8.

II. Rodríguez CA, Valiente ZC. Estrategias y resultados de la atención estomatológica a niños y adolescentes. Rev Cubana Estomatol.2003; 40 (1): 33-7.

12. Barber T. The concept of preventive Orthodontics. J Dent Chile. 2006; 12 (I). Consultado 03 de junio 2016.Disponible en: http://www.bus.sld.cu/revistas/ ord//2(2)/ord/0 I 297.htm

13. Onyeasso CO. Prevalence of malocclusion among adolescents in Ibadan, Nigeria. Am J Orthod Dentofacial Orthop. 2004; 126 (5): 604-7.

14. Di Rienzo J.A., Casanoves F., Balzarini M.G., Gonzalez L., Tablada M., Robledo C.W. InfoStat versión 2018. Grupo InfoStat, FCA, Universidad Nacional de Córdoba, Argentina. URL http://www.infostat.com.ar

15. Pérez Varela H, Ramos Morales C, Domínguez Fleites $L$. Tratamiento precoz de interferencias oclusales que provocan laterognatismo en niños de edades tempranas. Rev Cubana Ortod. I998; 30 (3): 63-8.
16. Urrego Burbano PA, Jiménez Arroyave LP, Londoño Bolívar MA, Zapata Tamayo M, Botero Mariaca P. Perfil epidemiológico de la oclusión dental en escolares de Envigado, Colombia. Rev Salud Pública. 20II [citado 12 Ago20 I4]; I3 (6). Disponible en: http:// www.revistas.unal.edu.co/index.php/revsaludpublica/article/view//9985/37886

17. Suárez Gómez L, Castillo Hernández R, Brito Reyes RD, Santana Méndez AT, Vázquez Monteagudo Y. Oclusión dentaria en pacientes con maloclusiones generales: asociación con el estado funcional del sistema estomatognático. Medicentro Electrónica [Internet]. 2018 Mar [citado 2018 Abr 23]; 22 (I): 5363. Disponible en:http:// scielo.sld.cu/scielo.php?script=sci_arttext \&pid $=S|029304320| 8000 \mid 00007 \&$ Ing=es. 